\title{
Probabilistic Real-Time Thermal Rating Forecasting for Overhead Lines by Conditionally Heteroscedastic Auto-Regressive Models
}

\author{
Fulin Fan, Student Member, IEEE, Keith Bell, Member, IEEE, and David Infield, Senior Member, IEEE
}

\begin{abstract}
Conventional approaches to forecasting of real-time thermal ratings (RTTRs) provide only single point estimates with no indication of the size or distribution of possible errors. This paper describes weather based methods to estimate probabilistic RTTR forecasts for overhead lines which can be used by a system operator within a chosen risk policy with respect to probability of a rating being exceeded. Predictive centres of weather conditions are estimated as a sum of residuals predicted by a suitable auto-regressive model and temporal trends fitted by Fourier series. Conditional heteroscedasticity of the predictive distribution is modelled as a linear function of recent changes in residuals within one hour for air temperature and wind speed or concentration of recent wind direction observations within two hours. A technique of minimum continuous ranked probability score estimation is used to estimate predictive distributions. Numerous RTTRs for a particular span are generated by a combination of the Monte Carlo method where weather inputs are randomly sampled from the modelled predictive distributions at a particular future moment and a thermal model of overhead conductors. Kernel density estimation is then used to smooth and estimate the percentiles of RTTR forecasts which are then compared with actual ratings and discussed alongside practical issues around use of RTTR forecasts.
\end{abstract}

Index Terms-Real-time thermal rating, Overhead lines, Probabilistic forecasting, Auto-regressive models, Fourier series, Continuous ranked probability score

\section{INTRODUCTION}

$\mathrm{T}$ HE real-time thermal rating (RTTR) of an overhead line $(\mathrm{OHL})$ is the maximum permissible level of power flow at which the OHL can be operated safely and reliably at the time in question [1]. The line current has to be limited under a certain value in order to avoid an excessive conductor temperature which leads to an unwanted acceleration of aging and excessive sag of a span which may violate the minimum required clearance [2]. An OHL is conventionally operated below a static line rating (SLR) which is estimated through a thermal model of overhead conductors [3], [4] using a

Manuscript received January 21, 2016; revised April 14, June 3, 2016. The work described in this paper is funded by the Scottish 'Energy Technology Partnership' (ETP), and Scottish Power Energy Networks (SPEN) and National Grid Electricity Transmission (NGET) through the Network Innovation Allowance.

The authors are with the Department of Electronic and Electrical Engineering, University of Strathclyde, Glasgow, G1 1XW, U.K. (e-mail: f.fan@strath.ac.uk; keith.bell@ strath.ac.uk; david.infield@strath.ac.uk). maximum allowable conductor temperature and a conservative set of weather conditions (high air temperature and low wind speed parallel to the span) for a particular season [5].

Instead of a conservative SLR, a RTTR system estimates or predicts the line's actual ampacity at a given time under prevailing weather conditions through monitoring or inference of the behaviour of overhead conductors. The additional headroom of an OHL's capacity exploited by a RTTR system can help network operators accommodate growth in power flow [6]. For investment planning timescales, RTTRs can be considered over a range of future operating conditions and, provided the operator has some reasonable measures available to them whenever power flows would exceed the real-time limits, can offer a cost-effective means to deal with power generation and demand growth or distributed generation connections that reduce the need for network reinforcement.

Weather-based RTTR forecasting techniques which use weather predictions are being widely developed for different horizons in operational planning and real-time system operation so as to analyse the electricity transmission congestion and to plan grid operation and make network capacity available to the energy market. Numerical weather forecasts and weather forecast ensembles provided by a weather service are usually used for estimating the point forecasts of RTTRs [7], [8] and the uncertainties of RTTR forecasts [2] respectively for a day ahead. Time series approaches have been employed in [9], [10] to derive predictive distributions of weather conditions for very few hours ahead from which random weather inputs were generated in a Monte Carlo procedure to estimate probabilistic RTTR forecasts.

This paper describes the use of conditionally heteroscedastic univariate auto-regressive (AR) and vector auto-regressive (VAR) models combined with a technique of minimum continuous ranked probability score (CRPS) estimation to estimate marginal distributions for air temperature $\left(T_{a}\right)$, wind speed $\left(w_{s}\right)$ and wind direction $\left(w_{d}\right)$ for 10 minutes (1 step) and half hour (3 steps) ahead based on historic 10-minute average time series. Numerous weather inputs are randomly sampled from each independent marginal distribution and then paired in order to have rank correlations similar to those among historic weather data. The RTTR forecasts are calculated based on numerous paired and unpaired weather inputs separately and their percentiles are smoothed and estimated by kernel density estimation. Point forecasts of solar radiation $\left(s_{r}\right)$ are used 
instead of probabilistic forecasts in this paper since, when wind speeds are above a particular, modest level [1] or when conductor temperatures are relatively high [11], conductor temperature is quite insensitive to the change in solar radiation. A flow chart describing the process of probabilistic RTTR forecasting is shown in Fig. 1.

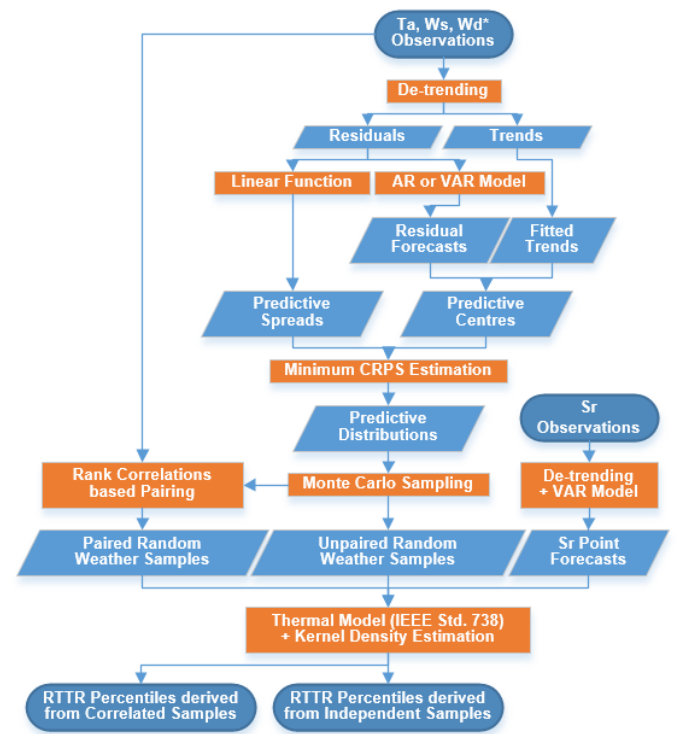

Fig. 1. Process of probabilistic RTTR forecasting (*Please refer to Section II describing the exact process of estimating predictive distribution of $w_{d}$ ).

The ideas are explored here in the context of a more than $90 \mathrm{~km}$ section of $132 \mathrm{kV}$ double circuit OHL in North Wales with a spur of around $10 \mathrm{~km}$. The 10-minute average weather data over 108 days, from 14/12/2012 to 31/03/2013, observed at 9 weather stations along the route were provided by Scottish Power Energy Networks from their project of "Implementation of real-time thermal ratings" (LCNF SPT1001) in North Wales [12]. A map of research area is shown in Fig. 2.

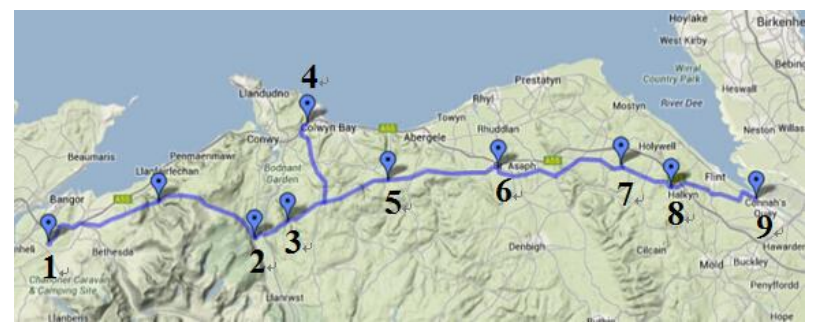

Fig. 2. Map showing the route of studied overhead line and locations of 9 weather stations in North Wales [12].

\section{Methodology}

\section{A. Temporal De-trending}

Statistical modelling generally requires the data to satisfy weak or second order stationarity. The inherent trends of non-stationary data may be misleading with regard to correlations among variables or the auto-correlation of a time series. Therefore, a de-trending method must be used to model and remove the temporal trends from the original data to ensure the stationarity of de-trended data from which model parameters are determined.

The temporal de-trending method applied here uses a Fourier series with the diurnal angular frequency of $2 \pi / 24 h$ [13] to extract the diurnal trends in the sliding training window, in which the training period consists of the observations in recent days at each weather station. The $3^{\text {rd }}$ and the $2^{\text {nd }}$ order Fourier series are used for solar radiation [14] and other weather parameters respectively.

\section{B. Univariate Auto-Regressive Model and Vector Auto-Regressive Model}

A univariate auto-regressive (AR) model of order $p$ estimates the forecast $\tilde{z}_{t}$ as a linear combination of $p$ historic values at a target location and a Gaussian noise term $e_{t}[15]$ :

$$
\tilde{z}_{t}=u+\sum_{i=1}^{p} \beta_{i} \tilde{z}_{t-i}+e_{t}
$$

where $\tilde{z}_{t}$ represents the deviation from the trend component. $u$ is a constant and $\beta_{i}$ are the auto-regressive parameters.

As an extension of a univariate AR model, the vector auto-regressive (VAR) model of order $p$, offers a way of producing the forecast as a weighted sum of historic time series not only at the target location but also from $(K-1)$ surrounding sampled locations [16]:

$$
\widetilde{\boldsymbol{Z}}_{t}=\boldsymbol{u}+\sum_{i=1}^{p} \boldsymbol{A}_{i} \widetilde{\boldsymbol{Z}}_{t-i}+\boldsymbol{E}_{t}
$$

where $\widetilde{\boldsymbol{Z}}_{t}$ is a $(K \times 1)$ vector consisting of $\tilde{z}_{t}$ at $K$ locations and $\boldsymbol{u}$ is a $(K \times 1)$ vector of constants. $\boldsymbol{E}_{t}$ is a $(K \times 1)$ vector of noise terms and $\boldsymbol{A}_{i}$ represents a $(K \times K)$ matrix of model coefficients at time lag $i$.

Equations (1) and (2) can be applied to air temperature, wind speed and solar radiation forecasting. We only consider the correlations between residuals during the daytime for solar radiation forecasting. Some changes in both equations are made for wind direction forecasting due to the circular properties of wind direction. Wind directions $\theta \in[-\pi, \pi)$ at each location are first decomposed along the easterly and northerly axes in the Cartesian coordinates as $\cos \theta$ and $\sin \theta$ which range between -1 and +1 respectively before de-trending and the application of the AR or VAR model. Thus, an AR model for wind direction forecasting can be regarded as a VAR model with two variables. Then the wind direction forecast is determined based on predictions of the easterly and northerly components. Please refer to [17] where the AR and VAR models are defined for wind direction forecasting.

\section{Predictive Probability Distribution}

Many users of forecasts, including power system operators, would like to know not only what the 'best' forecast is but also how wrong it might be, i.e. to have a probabilistic forecast that gives the probability of the true value of the forecasted quantity lying within a certain range. When producing a probabilistic forecast, the aim is to maximize the sharpness of predictive probability distributions subject to a calibration to minimize the uncertainty [18]. The calibration represents the statistical consistency between the predictive distributions and the observations [18]. The sharpness refers to the spread or concentration of the predictive distribution [18] which can be 
represented by the average width of central prediction intervals. Sharper or more concentrated predictive distributions are preferred under the constraint of calibration. The histogram of probability integral transform (PIT) is an effective tool to assess the calibration of probabilistic forecasts. In this case, the PIT is the value of the predictive cumulative distribution function (CDF) evaluated at the observation [19]. An approximately uniform PIT histogram reveals probabilistic forecasts to be nearly fully calibrated. The continuous ranked probability score (CRPS) value is a summary indicator to assess performance of probabilistic forecasting models with respect to the calibration and sharpness which will be detailed in section II.D.

Predictive probability distributions are usually assumed to be Gaussian [18]. Therefore, the predictive distribution of air temperature is taken to be normal denoted by $\mathrm{N}\left(\mu_{a}, \sigma_{a}\right)$. A truncated normal distribution with a cut-off at 0 denoted by $\mathrm{N}^{+}\left(\mu_{v}, \sigma_{v}\right)$ is used as the predictive distribution of wind speed due to its non-negativity [18]. In order to address the circular property of wind direction, the predictive distribution of wind direction is assumed to be von Mises denoted by $\operatorname{VM}\left(\mu_{\theta}, k\right)$ which is regarded as the circular analogue of the Gaussian distribution [20]. Their probability density functions (PDFs) can be found in [17].

The centres of predictive distributions can be modelled as a sum of residuals predicted by the AR or VAR forecasting models and the corresponding diurnal trends fitted by Fourier series. Conditional heteroscedasticity considers the predictive spread or concentration of predictive distribution to be time variable. The $L$-step-ahead predictive spread $\sigma_{t+L}$ or concentration parameter $k_{t+L}$ is modelled as a linear function of the root mean square of recent changes in residuals Res at the target location for air temperature and wind speed, assessed over 1 hour, as in (3). For wind direction, due to its circular nature, the concentration of recent observations at the target location is used, in this case over a period of 2 hours, as in (4). The concentration of recent wind direction observations, $k_{o}$, is calculated based on the code provided by Berens [21]. In this study, steps of 10 minutes are used to reflect the input data.

$$
\begin{gathered}
\sigma_{t+L}=c_{0}+c_{1}\left[\frac{1}{5} \sum_{j=0}^{4}\left(\operatorname{Res}_{t-j}-\operatorname{Res}_{t-j-1}\right)^{2}\right]^{\frac{1}{2}} \\
k_{t+L}=c c_{0}+c c_{1} k_{o}
\end{gathered}
$$

where $c_{0}, c_{1}, c c_{0}$ and $c c_{1}$ are non-negative coefficients. The experimental results obtained suggest that the selected lengths of 1 and 2 hours used to model the conditional heteroscedasticity result in an effective probabilistic forecasting model. The homoscedastic model, which assumes a constant spread or concentration, is also constructed as a comparison to analyse the advantages of the conditionally heteroscedastic model.

\section{Continuous Ranked Probability Score}

A technique of minimum continuous ranked probability score (CRPS) estimation proposed by Gneiting [22] is used to estimate the predictive probability distributions of weather variables. The CRPS is just one of the available scoring rules evolved from the Brier score and the ranked probability score, giving a numerical score to the event based on the difference between the predictive PDF $f$ and the observation $x_{o}$ [23]:

$$
\operatorname{crps}\left(f, x_{o}\right)=\int_{-\infty}^{\infty}\left[\int_{-\infty}^{x} f(y) d y-F_{o}\left(x, x_{o}\right)\right]^{2} d x
$$

where $F_{o}\left(x, x_{o}\right)$ is the Heaviside function and equal to 1 if the event that the percentile $x \geq x_{o}$ happens and 0 otherwise. The average value of crps, CRPS, used to assess probabilistic forecasts, should be minimized for probabilistic forecasting. For linear variables such as air temperature and wind speed, (5) can be written equivalently as [24]:

$$
\operatorname{crps}_{l}\left(F_{l}, x_{o}\right)=E\left\{\left|X-x_{o}\right|\right\}-\frac{1}{2} E\left\{\left|X-X^{\prime}\right|\right\}
$$

where $X$ and $X^{\prime}$ represent independent random samples from the linear predictive CDF $F_{l}$ and where $E\{\cdot\}$ is the expectation operator. The crps value for the normal distribution and the truncated normal distribution can be calculated directly based on the expressions derived by Gneiting which can be found in [22] and [18] respectively. The circular $\operatorname{crps}_{\mathrm{c}}$ for wind direction forecasting is estimated by using the angular distance $\alpha(\cdot)$ instead of the Euclidean distance in (6) [20]:

$$
\operatorname{crps}_{c}\left(F_{c}, \theta_{o}\right)=E\left\{\alpha\left(\Theta, \theta_{o}\right)\right\}-\frac{1}{2} E\left\{\alpha\left(\Theta, \Theta^{*}\right)\right\}
$$

where $\Theta$ and $\Theta^{*}$ represent the independent randomly sampled wind directions from the circular predictive distribution function $F_{c}$. The term $\theta_{o}$ is the observed wind direction. The first term on the right-hand side of (7) can be expressed as:

$$
E\left\{\alpha\left(\Theta, \theta_{o}\right)\right\}=\frac{1}{2 \pi I_{0}(k)} \int_{-\pi}^{\pi} \alpha\left(x_{\theta}, \theta_{o}\right) e^{k \cos \left(x_{\theta}-\mu_{\theta}\right)} d x_{\theta}
$$

where $I_{0}(\cdot)$ represents the modified Bessel function of the first kind of order zero [20] and $\mu_{\theta}$ is the predictive centre of von Mises distribution. It is found that $E\left\{\alpha\left(\Theta, \theta_{o}\right)\right\}$ is only dependent on the concentration parameter $k$ and the angular distance between $\theta_{o}$ and $\mu_{\theta}$. A look-up table for $E\left\{\alpha\left(\Theta, \theta_{o}\right)\right\}$ in terms of both $k$ with accuracy of 0.1 and $\alpha\left(\theta_{o}, \mu_{\theta}\right)$ with accuracy of $0.0017\left(0.1^{\circ}\right)$ can be built up in order to reduce computation time due to iterative calculation for the determination of model parameters. The second term on the right-hand side of (7) depends on $k$ only. A look-up table is also built for the second term $\frac{1}{2} E\left\{\alpha\left(\Theta, \Theta^{*}\right)\right\}$ in terms of $k$ with accuracy of $0.0017\left(0.1^{\circ}\right)$ according to the procedure in [20].

The parameters in the AR or VAR forecasting models and coefficients representing the predictive spread or concentration parameter are estimated with the objective of minimizing the average value of $\operatorname{crps}_{l}$ or $\operatorname{crps}_{c}$. Initial values of the AR or VAR parameters are determined from the de-trended data at each location using least squares estimation [25]. Initial values of the non-negative coefficients modelling the spread or concentration parameter are set to be 0.1 and 1.0 respectively. 
The dependencies of the probability distribution of wind direction, $E\left\{\alpha\left(\Theta, \theta_{o}\right)\right\}$ and $\frac{1}{2} E\left\{\alpha\left(\Theta, \Theta^{*}\right)\right\}$ on concentration parameter $k$ have been explored and there is little change for $k$ over 200. Therefore, $k$ is limited to a maximum value of 200 .

\section{E. Correlating Weather Input Variables for RTTR Estimations}

The thermal balance of an overhead conductor is kept with heat generated by Joule heating and solar heating, and heat lost by convection and radiation from the conductor surface. The steady-state or continuous RTTR would lead to a maximum allowable conductor temperature for specific weather conditions under the assumption of the conductor being in thermal equilibrium [3].

The rating of an overhead conductor is influenced by different weather parameters in a complex way. It is difficult to exactly calculate the probabilistic RTTR forecasts from predictive distributions of weather parameters through a deterministic thermal model of overhead conductors [3]. In this case, Monte Carlo simulation [26] allows us to model different combinations of weather input variables, as well as their interdependent relationships. It is used to produce a large number $N_{M C}$ of sampled values of steady-state RTTR forecasts by evaluating the outputs (ratings) of the thermal model of overhead conductors [3] for inputs of values randomly sampled from the modelled predictive probability distributions of air temperature, wind speed and wind direction and the point forecasts of solar radiation.

Random input variables from marginal normal, truncated normal and von Mises distributions are produced through the codes provided in [27], [28] and [21]. These random weather input variables can be regarded as being independent of each other since the results showed that their correlations were smaller than 0.01 when $N_{M C}$ equals $10^{4}$. Then, a rank correlation based pairing method [29], regardless of the type of marginal distribution, is adopted to pair independent random weather input variables so as to have a correlation similar to that calculated from the weather observations in recent days.

The evaluations of the Spearman's rank correlation coefficient between linear variables and the $\mathrm{C}$-association describing the rank relationship between a linear variable and a circular variable have been detailed in [9] and [30]. Then, a rank correlation matrix $C$ of size $(3 \times 3)$ can be computed from each pair of recent weather observations. We generate a new matrix $M$ of size $\left(N_{M C} \times 3\right)$ in which each column consists of arbitrary van der Waerden scores $\Phi^{-1}\left(i /\left(N_{M C}+1\right)\right), i=1, \ldots, N_{M C}[29]$, where $\Phi(\cdot)$ represents the $\mathrm{CDF}$ of a standard normal distribution. The lower triangular matrices $P$ and $Q$ are obtained through Cholesky factorization such that $P P^{\prime}=C$ and $Q Q^{\prime}=T$ where $T$ is the sample correlation matrix associated with $M$. The matrix $M^{*}=$ $M\left(P Q^{-1}\right)^{\prime}$ would have a rank correlation matrix quite similar to $C$. The random weather input variables generated from each independent marginal distribution are then sorted according to the order of corresponding column in $M^{*}$ [29]. In this manner, the dependence among weather data is incorporated into paired multivariate random weather input variables.

\section{RESUltS AND MODEl VALIDATION}

The mathematical calculations included in this paper are all accomplished using MATLAB [31]. We will detail the process of estimating $L$-step-ahead forecasts of steady-state RTTRs. As was noted above, we have access to weather data every 10 minutes; the examples given therefore use step lengths of 10 minutes. Although the first challenge is to develop reasonable forecasts for 1 step ahead, in practice, it is very difficult for system operators to make use of 10-minute forecasts as updates of system state from an energy management system and implementation of any action required to secure the system would typically take at least that long. Although the notice given to operators would ideally be much longer than half-an-hour, as a means of establishing the viability of the described approach and giving something of at least some value to system operators, we also present results for 3 steps ahead.

\section{A. AR and VAR Model Validation Procedure}

The orders of the AR and VAR models can be determined by the inspection of partial autocorrelation functions [15] or the comparison of forecast errors for different model orders [13], [32]. The AR and VAR model point forecast performance is assessed in terms of root mean square error (RMSE). The length of sliding training window, used to model diurnal trends and determine auto-regressive parameters, is determined as that which gives the best improvement in RMSE over persistence forecasting which supposes that forecasts in the future are equal to present values [33]. The model parameters are updated once a day which has been proved in the tests conducted here to be sufficient to give accurate forecasts. In order to determine the window lengths, we initially assume an order of 2 for both AR and VAR models based on Hill's work [13] in which varying the order from 2 to 4 produced less than 1\% improvement in RMSE of 1-step-ahead forecasts of hourly wind speed. As an illustration, the improvement over persistence in RMSE for $\operatorname{AR}(2)$ and $\operatorname{VAR}(2)$ models with varying training window length for each weather parameter is tested for all stations and the results at station 2 are shown in Fig. 3.
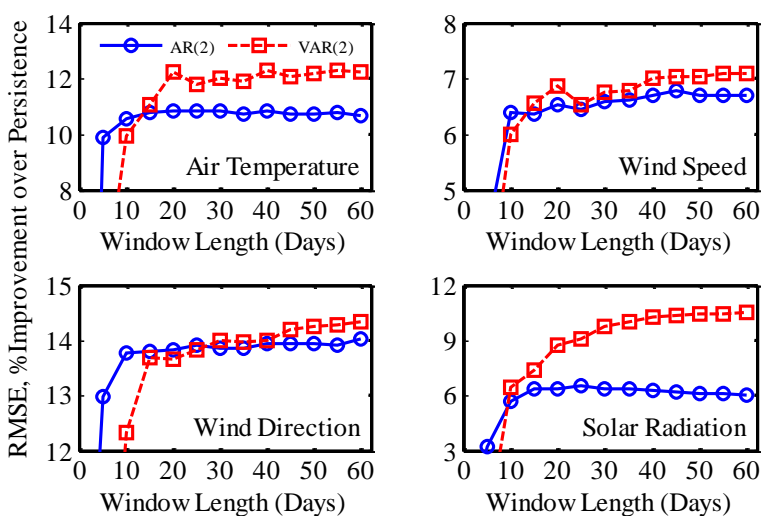

Fig. 3. Improvement over persistence in RMSE for 1 step ahead for AR(2) and VAR(2) models with varying training window length for each weather parameter at station 2 .

The experimental results demonstrate that (a) given a sufficiently long training window, VAR models perform better than AR models; (b) although there will be a range of effective 
training window lengths, for AR and VAR models here 40, 45, and 45 days are reasonable choices respectively for air temperature $\left(T_{a}\right)$, wind speed $\left(w_{s}\right)$ and wind direction $\left(w_{d}\right)$ since these selected lengths have been found to work well for all stations; and (c) for solar radiation $\left(s_{r}\right), 25$ and 45 days should be selected for AR and VAR models respectively.

In order to confirm the models' orders the weather forecasts for 1 step ahead at station 2 produced by the AR and VAR models of different orders $p$ are compared with persistence forecasts, as shown in Fig. 4. Performing better than AR models, the VAR(1) model is used to predict $s_{r}$ due to insignificant improvement using higher orders. For other weather parameters, less than $1 \%$ improvements are achieved when orders are over 2. Furthermore, the VAR model is mostly shown to give a lower RMSE than the AR model of a same order due to the additional capture of the inherent spatial correlations among the field data [13], [32]. However, the AR(4) models having fewer auto-regressive parameters perform similarly to or even better than the VAR(2) models for $w_{s}$ and $w_{d}$ forecasting at all weather stations. Due to the fact that having fewer parameters reduces computation time in the process of minimizing CRPS value, the AR(4) models are preferred for $w_{s}$ and $w_{d}$ rather than the $\operatorname{VAR}(2)$ models. A VAR(2) model is adopted to estimate 1 -step-ahead $T_{a}$ forecasts.
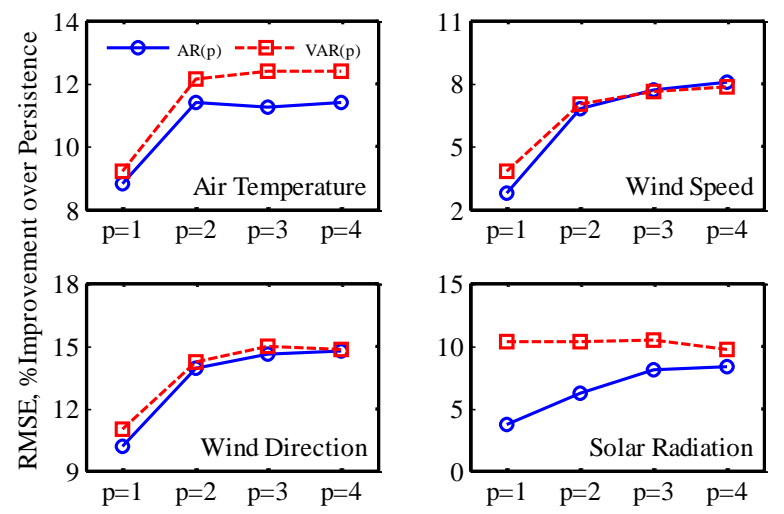

Fig. 4. Improvement over persistence in RMSE for 1 step ahead for AR and VAR models of different orders for each weather parameter at station 2.

The aforementioned procedures are carried out to validate the AR and VAR models for 3-step-ahead forecasting and the $\operatorname{VAR}(2), \operatorname{AR}(4), \operatorname{AR}(6)$ and $\operatorname{VAR}(1)$ models with their corresponding training windows of $40,45,45$ and 45 days are employed respectively for $T_{a}, w_{s}, w_{d}$ and $s_{r}$ in this case.

\section{B. Assessment of Probabilistic Weather Forecasts}

As was noted in sections II.C and II.D, the sharpness or spread of a predictive distribution can be indicated by the average width of central predictive intervals (CPIs) and the continuous ranked probability score (CRPS) value is a summary metric designed to reflect both the sharpness and calibration. Small values are sought for each. Probabilistic 1-step-ahead weather forecasts generated by the four models, homoscedastic $\mathrm{AR}(4)-\mathrm{H}$ and $\operatorname{VAR}(2)-\mathrm{H}$ models and conditionally heteroscedastic AR(4)-CH and VAR(2)-CH models, are assessed as shown in Fig. 5 which indicates the improvement over the AR(4)-H model in CRPS and average widths of 50\% CPIs for the other three probabilistic models.
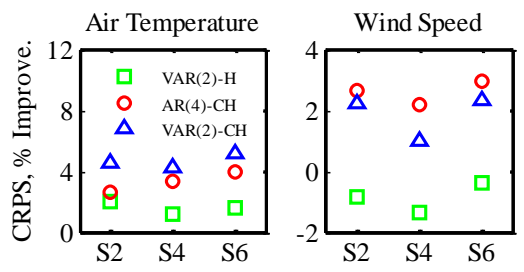

Wind Direction
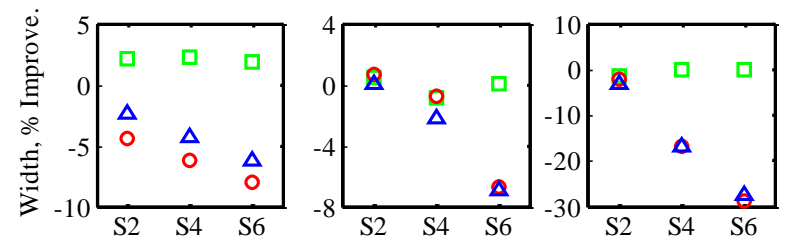

Fig. 5. Improvement over the AR(4)-H model in CRPS and average widths of $50 \%$ CPIs for probabilistic 1-step-ahead weather forecasts for the VAR(2)-H, $\mathrm{AR}(4)-\mathrm{CH}$ and VAR(2)-CH models at stations 2, 4 and 6.

It is found that over half of the time the predictive distributions modelled by conditionally heteroscedastic models are more concentrated than the distributions modelled by homoscedastic models, e.g. AR(4)-CH model spreads for wind speed at station 4 being smaller for around $58.2 \%$ of the time. However, due to some extremely dispersive distributions for conditionally heteroscedastic models, most of the average widths of 50\% CPIs modelled by homoscedastic models are smaller on average as shown in Fig. 5, at the cost of losing a certain calibration which could be inspected from their PIT histograms [17]. The calculated CRPS values suggest that the VAR(2)-CH, AR(4)-CH, and AR(4)-CH models should be employed to estimate probabilistic 1-step-ahead forecasts for $T_{a}, w_{s}$ and $w_{d}$ respectively. For probabilistic 3-step-ahead forecasting, the $\operatorname{VAR}(2)-\mathrm{CH}, \mathrm{AR}(4)-\mathrm{CH}$ and $\mathrm{AR}(6)-\mathrm{CH}$ models are selected for each weather parameter. The $50 \%$ and 90\% CPIs associated with their 1-step-ahead predictive centres for $w_{s}$ and $w_{d}$ modelled by the $\mathrm{AR}(4)-\mathrm{CH}$ models on 27/03/2013 at station 4 are plotted in Fig. 6.
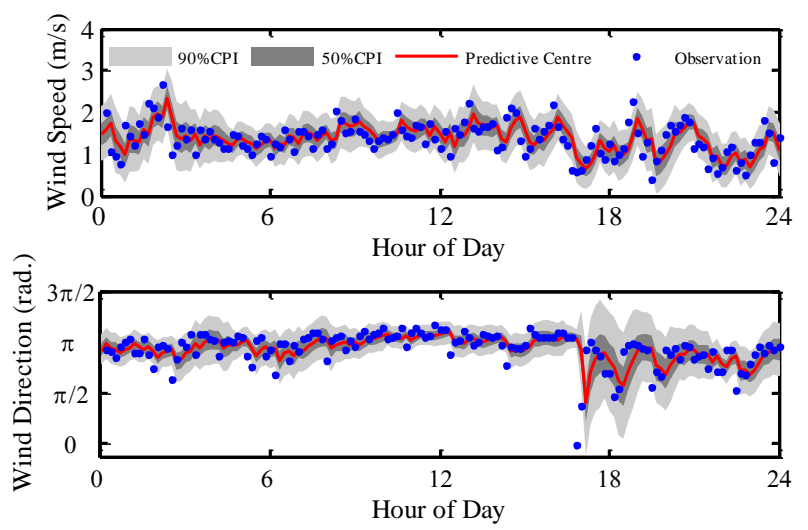

Fig. 6. The 50\% and 90\% CPIs associated with 1-step-ahead predictive centres for $w_{s}$ and $w_{d}$ modelled by the AR(4)-CH models on 27/03/2013 at station 4.

The experimental results indicate that the $w_{s}$ and $w_{d}$ observations are located within the 50\% CPIs for around 53.2\% and $51.1 \%$ of the time respectively and within the $90 \%$ CPIs for around $88.6 \%$ and $86.6 \%$ of the time respectively. In addition, the 1-step-ahead point forecasts or expected values of $w_{s}$ and $w_{d}$ 
estimated by the AR(4)-CH models having RMSEs of 0.38 $(\mathrm{m} / \mathrm{s})$ and 0.68 (radians) respectively give $7.7 \%$ and $10.4 \%$ improvements over persistence forecasting at station 4.

\section{Assessment of Probabilistic RTTR Forecasts}

The probabilistic 1-step-ahead and 3-step-ahead steady-state RTTR forecasts at two spans CQ34-CQ35 and AC102-AC101B in proximity to stations 4 and 6 are studied. They are composed of 'Lynx' ACSR $175 \mathrm{~mm}^{2}$ and 'Poplar' AAAC $200 \mathrm{~mm}^{2}$ conductors with maximum allowable conductor temperatures of $50^{\circ} \mathrm{C}$ and $75^{\circ} \mathrm{C}$ which are reduced to $45^{\circ} \mathrm{C}$ and $70^{\circ} \mathrm{C}$ respectively for reasons of conservatism [12].

Different pairs of weather variables are correlated. In the Monte Carlo process, a large number $N_{M C}=10^{4}$ of random weather input variables sampled independently from predictive distributions modelled by the conditionally heteroscedastic models at a particular future time, are paired based on the rank correlations computed from recent weather observations, i.e. within 15 days. The paired random samples of air temperature and wind speed having rank correlations quite close to those between their recent observations are shown in Fig. 7.
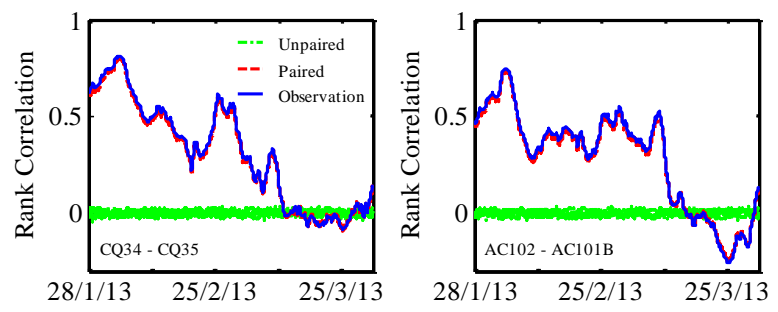

Fig. 7. Rank correlations between unpaired, paired random samples of air temperature and wind speed and their recent observations within 15 days.

The numerous correlated and independent random weather inputs at a particular future moment are separately used to calculate the possible RTTR forecasts from which a sample cumulative distribution function (CDF) can be extracted. The percentiles of calculated RTTR forecasts can then be smoothed and estimated by kernel density estimation [34]. The PIT histograms of probabilistic steady-state RTTR forecasts for 3 steps ahead generated by the conditionally heteroscedastic $(\mathrm{CH})$ models based on correlated ( $\mathrm{CH}-\mathrm{C})$ weather inputs, the $\mathrm{CH}$ models based on independent (CH-I) weather inputs, and the homoscedastic $(\mathrm{H})$ models based on independent $(\mathrm{H}-\mathrm{I})$ weather inputs for two spans are plotted in Fig. 8 respectively. The relative frequency of 0.01 per percentile for a uniformly distributed PIT histogram is denoted by a black solid line.

The PIT histograms of RTTR forecasts derived from $\mathrm{CH}$ models are shown to have a better calibration than those derived from $\mathrm{H}$ models. The hump shaped H-I PIT histograms indicate that the H-I probabilistic RTTR forecasts are over-dispersive. Furthermore, the relative frequencies at both ends of the CH-C PIT histograms are high which reveals that the predictive distributions of RTTRs are less dispersive. This might be due to the long-term positive correlations between air temperature and wind speed as shown in Fig. 7. The increased cooling effect induced by high wind speeds is usually reduced by accompanied high air temperatures, and vice versa.
Therefore, the widths from the $1^{\text {st }}$ to $99^{\text {th }}$ percentiles of $\mathrm{CH}-\mathrm{C}$ probabilistic RTTR forecasts are commonly smaller than those of CH-I probabilistic RTTR forecasts. The significant deviations from the ideal relative frequency of 0.01 at both ends of the CH-C PIT histograms are mitigated in the CH-I PIT histograms. In theory, the independent random weather inputs should be treated as correlated. However, the additional correlations aggravate the concentration of under-dispersive CH-I probabilistic RTTR forecasts in this work. Through checking the linear correlation between PIT values of the probabilistic RTTR forecasts for each of the weather predictions, wind speed is found to be the dominant factor affecting the distributions of the PIT histograms of RTTR forecasts. The PIT histograms of probabilistic RTTR forecasts for 1 step ahead are similar to the histograms for 3 steps ahead derived from the same models. The conclusions obtained from the histograms for half hour ( 3 steps) ahead can also be summarized from the histograms for 10 minutes (1 step) ahead.

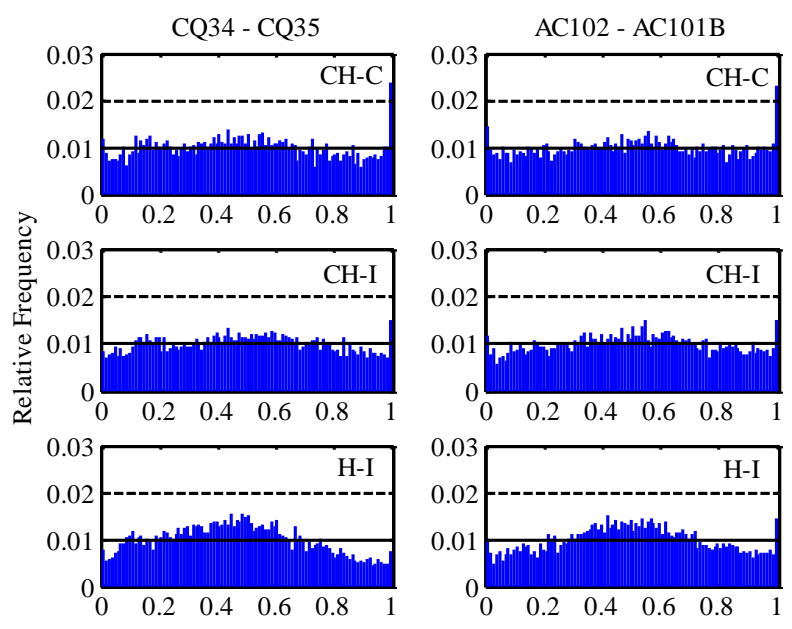

Fig. 8. PIT histograms of 3-step-ahead probabilistic steady-state RTTR forecasts for CQ34-CQ35 and AC102-AC101B.

Fig. 9 and Fig. 10 show the ratios of $5^{\text {th }}-95^{\text {th }}$ percentiles, $25^{\text {th }}-75^{\text {th }}$ percentiles, point forecasts of steady-state CH-I RTTRs for 1 step ahead and 3 steps ahead and weather observation based RTTRs to the static line ratings (SLRs) on 27/03/2013 for CQ34-CQ35 and AC102-AC101B respectively. The SLRs for the two spans are 485A and 607A in winter (Dec., Jan. and Feb.) and 450A and 581 A in spring (Mar.) respectively [12]. The distributions of 3-step-ahead RTTR forecasts are less concentrated than that of 1-step-ahead forecasts on average due to the fact that we want to preserve a satisfactory calibration while the forecast errors increase for RTTRs for 3 steps ahead.

In spite of the extra current-carrying capacity released by RTTRs being several times higher than the SLRs for most of the time, in practical application, the upgrading of ratings will usually be limited to levels around $25 \%$ above the SLRs to prevent the protection scheme tripping and to reflect the constraints of other circuit equipment [35], [36]. The accuracies (RMSEs) of RTTR forecasts estimated by the CH-I models for the three levels of less than 100\% SLR, 100-125\% SLR and above $125 \%$ SLR, are estimated respectively as tabulated in 
Table I. It may be noted that the point forecasts of steady-state RTTRs derived from the correlated and independent weather samples are quite similar.

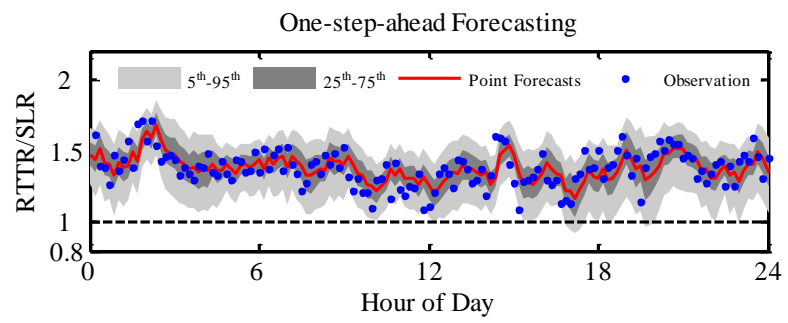

Three-step-ahead Forecasting

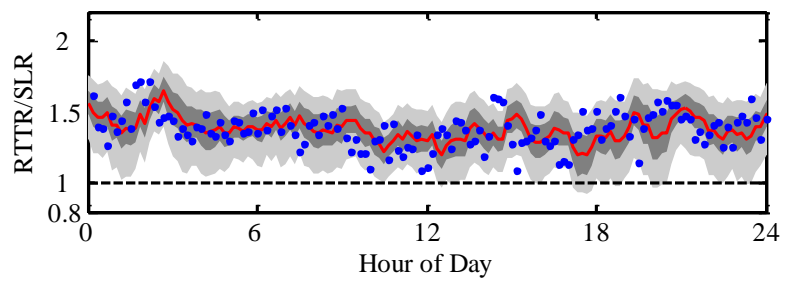

Fig. 9. Probabilistic 1-step-ahead and 3-step-ahead steady-state RTTR forecasts on 27/03/2013 for CQ34-CQ35.

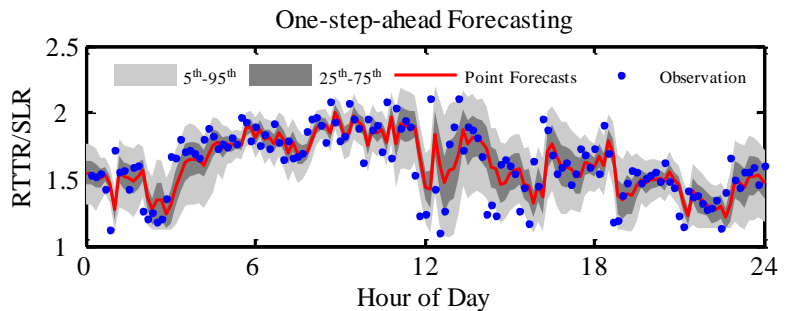

Three-step-ahead Forecasting

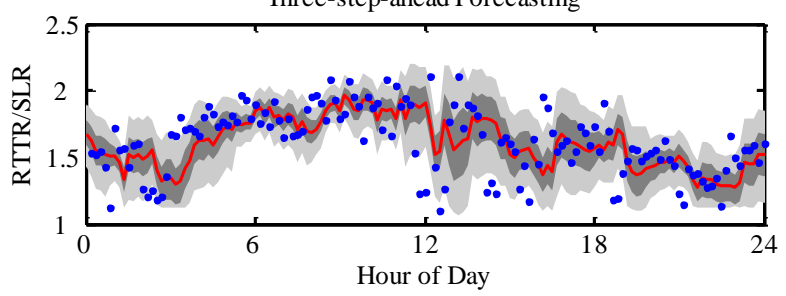

Fig. 10. Probabilistic 1-step-ahead and 3-step-ahead steady-state RTTR forecasts on 27/03/2013 for AC102-AC101B.

TABLE I

RMSE (A) OF RTTRS FOR CH-I MODELS FOR THE THREE LEVELS OF IMPROVEMENT OF SLRS AND THEIR IMPROVEMENT (\%) OVER PERSISTENCE

\begin{tabular}{c|c|c|c|c|c}
\hline \hline \multicolumn{2}{c|}{ Span } & \multicolumn{2}{c|}{ CQ34-CQ35 } & \multicolumn{2}{c}{ AC102-AC101B } \\
\hline \multicolumn{2}{c}{ Step(s) ahead } & 1 & 3 & 1 & 3 \\
\hline \multirow{2}{*}{ Total } & RMSE & 53.3 & 61.7 & 65.1 & 77.6 \\
\cline { 2 - 6 } & Improve. & $11.34 \%$ & $12.07 \%$ & $9.25 \%$ & $11.46 \%$ \\
\hline$\leq 100 \%$ & RMSE & 80.7 & 95.1 & N/A & N/A \\
\cline { 2 - 6 } SLR & Improve. & $-7.51 \%$ & $-5.03 \%$ & N/A & N/A \\
\hline $100-125 \%$ & RMSE & 52.9 & 59.1 & 89.9 & 113.6 \\
\cline { 2 - 6 } SLR & Improve. & $17.45 \%$ & $18.59 \%$ & $-4.25 \%$ & $-2.56 \%$ \\
\hline$>125 \%$ & RMSE & 48.6 & 57.0 & 61.0 & 71.6 \\
\cline { 2 - 6 } SLR & Improve. & $12.95 \%$ & $13.42 \%$ & $12.21 \%$ & $14.95 \%$ \\
\hline \hline
\end{tabular}

${ }^{\dagger}$ Only one rating observation was found below SLR for AC102-AC101B.

The CH-I model predictions are shown to be significantly better than persistence. If the practical limit for uprating is $125 \%$ of SLR, the results suggest that an uprating of $125 \%$ can be applied with reasonable confidence when the forecast in respect of thermal balance on the OHL conductor suggests a possible rating of more than $125 \%$. For the key level of $100-125 \%$ SLR, the $\mathrm{CH}-\mathrm{I}$ models perform much better for CQ34-CQ35 but worse for AC102-AC101B than persistence. That is, the CH-I forecasting models perform worse for both spans at the levels of their respective lower ratings (the levels below SLR for CQ34-CQ35 and of 100-125\% SLR for AC102-AC101B). It is found that wind speed observations corresponding to lower rating levels are mainly distributed within the ranges of smaller values where the improvement in RMSE over persistence for $\mathrm{AR}(4)-\mathrm{CH}$ models is quite small, i.e. 0.66\% at CQ34-CQ35 and $0.27 \%$ at AC102-AC101B. The distributions of errors of 3-step-ahead steady-state RTTR forecasts at the level below the SLR and the distributions of corresponding wind speed and wind direction observations and their 3-step-ahead forecasts for CQ34-CQ35 are compared as shown in Fig. 11.

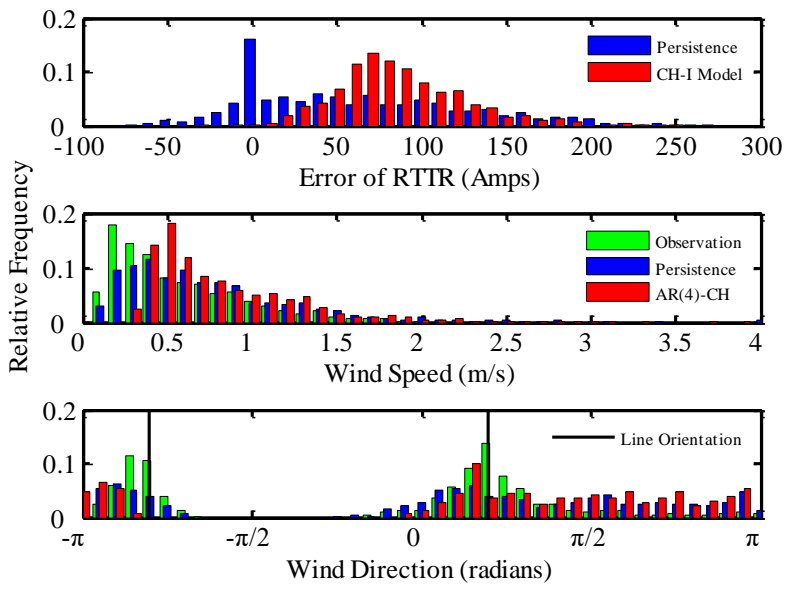

Fig. 11. Distributions of errors of 3-step-ahead steady-state RTTR forecasts, the wind speed and direction observations and their 3-step-ahead forecasts corresponding to the rating level below the SLR for CQ34-CQ35.

Fig. 11 reveals that the RTTRs predicted by the $\mathrm{CH}-\mathrm{I}$ model are overestimated at the low rating levels $(<\mathrm{SLR})$ due to the wind speed forecasts being significantly overestimated by the $\mathrm{AR}(4)-\mathrm{CH}$ model. It is interesting that a significant proportion of the RTTR errors by the persistence forecasting are concentrated around zero which might be due to the predicted and observed wind speeds having very low values $(\leq 0.4 \mathrm{~m} / \mathrm{s})$. This will lead to small differences between the predicted and observed RTTRs, especially when wind directions are nearly parallel to the overhead span which mitigates wind cooling. The experimental results indicate that, at this low rating level, the persistence forecasts and observations of wind speed are both under $0.4 \mathrm{~m} / \mathrm{s}$ for $23.6 \%$ of the time and both under $0.3 \mathrm{~m} / \mathrm{s}$ for $10.3 \%$ of the time, while for the AR(4)-CH model, the corresponding percentages of time are $6.5 \%$ and $0.3 \%$ respectively. The AR(4)-CH model's overestimation at lower values of wind speed can also explain the unsatisfactory performance of the CH-I model at the level of 100-125\% SLR for AC102-AC101B. Therefore, the lower RTTR percentiles should be applied so as to avoid the risk of using the overestimated point forecasts of RTTRs.

The average additional capacities (AAC) above SLR (in \%) released by the CH-I $1^{\text {st }}, 5^{\text {th }}$ and $10^{\text {th }}$ percentiles for 3 steps ahead and the percentage of time that these percentiles are 
above SLR are listed in Table II. The extra thermal headroom which can be exploited by lower percentiles for AC102-AC101B is much higher than that for CQ34-CQ35.

TABLE II

THE AVerage AdDitional CAPACITIES (AAC) ABOVE SLR (\%) FOR CH-I $1^{\text {sT }}$, $5^{\text {TH }}$ AND $10^{\text {TH }}$ PERCENTILES FOR 3 STEPS AHEAD AND THE PERCENTAGE OF TIME FOR THE PERCENTILES ABOVE SLR

\begin{tabular}{c|c|c|c|c|c|c}
\hline \hline & \multicolumn{2}{|c|}{$1^{\text {st }}$ Percentiles } & \multicolumn{2}{c|}{$5^{\text {th }}$ Percentiles } & \multicolumn{2}{c}{$10^{\text {th }}$ Percentiles } \\
\hline$\%$ above SLR & AAC & Time & AAC & Time & AAC & Time \\
\hline CQ34-CQ35 & $3.5 \%$ & $44.5 \%$ & $10.7 \%$ & $59.3 \%$ & $15.4 \%$ & $68.7 \%$ \\
\hline AC102-AC101B & $24.9 \%$ & $99.2 \%$ & $31.6 \%$ & $99.8 \%$ & $35.9 \%$ & $99.9 \%$ \\
\hline \hline
\end{tabular}

For the sake of having unambiguous limits to system operation that, in turn, drive decisive action, a system operator is likely to set a policy in which a particular percentile is adopted from a probabilistic forecast and regarded as The Limit. This is consistent with current practice in Britain in which static line ratings are calculated based on a certain small probability of the actual rating being greater than the quoted SLR [37]. One reasonable policy that might be adopted would be to ensure that the loading on a line never exceeds the $5^{\text {th }}$ percentile (P5 value) from the probabilistic forecast. The effectiveness of any policy should be checked. Fig. 12 shows the differences between the $5^{\text {th }}$ percentile of the 3 steps ahead forecast and the actual rating when the time comes. If the probabilistic forecasting works well then, on average, the actual rating should be less than the P5 forecast in no more than 5\% of cases.

As noted above, various practical issues will prevent more than a certain uprating relative to the SLR, e.g. $25 \%$. As a consequence, Fig. 12 shows two sets of two results for two different OHL spans: for 6662 and 4207 cases in which the P5 forecast was less than $125 \%$ of SLR; and 2410 and 4865 cases in which it was greater than or equal to $125 \%$. For CQ34-CQ35, the test set showed $3.68 \%$ of the former cases in which the actual rating was less than the P5 value and $5.02 \%$ of the latter. For AC102-AC101B, the actual rating was less than the P5 forecast in $3.02 \%$ of cases when the P5 forecast rating was less than $125 \%$ of SLR and $5.32 \%$ otherwise. Although these seem reasonable results, the test set contains an example of a quite large positive difference between the P5 forecast and the actual rating on AC102-AC101B: 262.6A compared with the relevant seasonal SLR of 607A. This occurred after a period of 6 hours in which the wind speed had been greater than $3 \mathrm{~m} / \mathrm{s}$ which led to a forecast wind speed also of greater than $3 \mathrm{~m} / \mathrm{s}$ but where it turned out actually to be $1.3 \mathrm{~m} / \mathrm{s}$. In addition, at that moment, the wind blew at $45^{\circ}$ to the span whereas it had previously been perpendicular to it. However, regardless of how much above $125 \%$ the P5 forecast was, if the system operator limited loading on the line to no more than $125 \%$, such a limit would have proved to be too high relative to the actual rating in only $3 \%$ and $2.1 \%$ of cases for the different spans and the extreme case noted would have presented no problem.

It can be seen from Fig 12 that the standard deviation of differences is higher for cases forecasting a small uprating than for those that forecast a higher uprating. This can be explained by the precise uprating being more sensitive to the exact wind speed at low speeds than at high wind speeds.

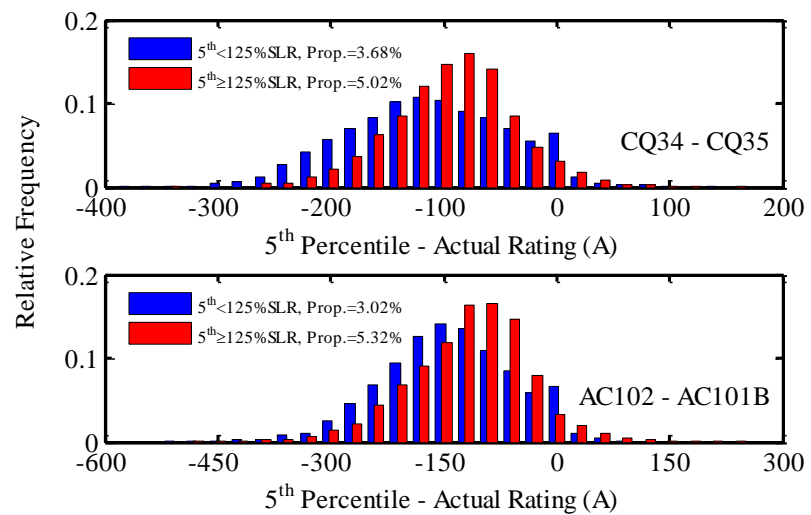

Fig. 12. The distributions of differences between the $5^{\text {th }}$ percentile forecasts for 3 steps ahead and their corresponding actual ratings and the proportion of positive differences for cases (a) when $5^{\text {th }}$ percentile forecast is less than $125 \%$ of SLR and (b) when it is greater than or equal to $125 \%$.

\section{DISCUSSION}

System operators will tend to be risk averse and incur the penalty of, for example, higher congestion costs rather than be exposed to any significant probability of a conductor's temperature exceeding its limit [38]. The lower percentiles of RTTR forecasts are therefore more interesting and useful for system operators in the dispatch of power flows. Here we adopt the predictive RTTR percentiles modelled by the independent random weather inputs sampled from the conditionally heteroscedastic forecasting models which have a good calibration at lower percentiles. In addition, the estimated lower percentiles are conservative due to their relative frequencies being slightly smaller than 0.01 .

In practice, although heat transfer around each span of an overhead line might suggest possible uprating of $100 \%$ or more relative to the seasonal static rating, other considerations preclude this, not least the settings of protection on the circuit. These might limit the practical uprating that can be applied to somewhere in the order of $25 \%$ [35], [36].

The work reported here has examined forecast horizons of 30 minutes (three 10-minutes time steps). Given time for SCADA measurements to be received, a system state estimation to be updated and some consideration of the implications of the system's state relative to prevailing and anticipated ratings, this is sufficient time for a system operator to take action based on the forecast result. However, actions that might be taken to reduce loading if the forecast real-time rating would otherwise be exceeded are, in effect, limited to generation re-dispatch, most obviously curtailment of output at or near the sending end of the critical line, or some demand reduction in the vicinity of the receiving end, e.g. where possible, by switching demand to another substation through distribution network reconfiguration. (Where this is not possible, to avoid interruptions to demand that have not been contracted in advance, it might only be possible to effect modest reductions through changes to voltage targets within the distribution network.)

Longer notice periods would open up other courses of action. This includes increases in generation output to back off the transfer of power on the critical line, especially if sufficient 
availability and 'headroom' for the option had been procured in advance. If emergency return to service times permit it, another possibility is recall of any planned network outages that would be effective in relieving the loading of the critical line. Beyond around 6 hours, forecasting would most likely depend on use of numerical weather prediction models by specialist meteorologists rather than statistical methods, though statistics can help in the evaluation of ensemble forecasts.

In the longer-term, one of the benefits of real-time thermal ratings should be to allow enhanced utilisation of particular network assets and, as a consequence, reduced need for additional assets. This should be assessed on a risk basis having in mind the courses of action available when power transfers exceed the real-time rating, the probability of those actions being required and their cost. The availability of such actions and, in the short-term, the availability of data to inform investment decisions, may limit the range of contexts in which real-time ratings can be implemented. However, the case study given in this paper provides an example of a context in which it should be possible to make full use of RTTR. The double circuit in North Wales provides not only a route for export of power from the western end but also a course of action when a rating would be exceeded, which is reduction of generation output. Moreover, it is likely to be required only when an unplanned circuit outage occurs. Given a forecast not only of continuous ratings but also short-term, post-fault ratings (which is the subject of further work), pre-fault curtailment of generation output should rarely be required with the obvious benefit of maximising utilisation of the available generation.

A further practical consideration is validation of both real-time and forecast ratings. Ideally, especially if a circuit is highly critical or its loss from service would have a large impact, some form of monitoring technology should be available to compare calculated ratings with what the monitoring suggests they actually are. A range of technologies is discussed in [6].

If, as has been discussed, the forecasting methodology is such that the forecast percentiles can be regarded with confidence, they then give a probability of the actual rating being lower than the value being assumed by the system operator. The choice of percentile should be informed by the impact of exceedance. In a worst case, the line might trip though, if the system was being operated to be $\mathrm{N}-1$ secure, in practice a step change in loading would be observed following a trip and the conductor temperature would not rise to the acceptable maximum instantaneously. A number of possible corrective actions have been discussed above. Detailed analysis of a range of possible conditions would reveal the time available for action, the possible need for automated actions and inform the choice of percentile that should be adopted.

\section{CONCLUSIONS AND FUTURE WORK}

This paper has proposed a weather based approach to probabilistic real-time thermal rating (RTTR) forecasts for overhead lines (OHLs) based on conditionally heteroscedastic auto-regressive (AR-CH) forecasting models. The proposed methods have been tested on two spans composed of different types of overhead conductors. The RTTR percentiles for a particular span derived from independent random weather samples generated from the $\mathrm{CH}$ models are preferred due to their good calibration. The correlations added into random weather samples narrow the predictive distributions of RTTRs so that the distributions of RTTR forecasts derived from paired random weather samples become more concentrated.

The RTTR point forecasts estimated by the probabilistic RTTR forecasting model have been shown to be significantly better than persistence for both 10 minutes (1 step) and half hour (3 steps) ahead. However, the overestimation of wind speed forecasts at lower values leads to an unsatisfactory performance of RTTR forecasting at low rating levels. In practice, a risk averse system operator is likely to adopt a policy in which there is a small probability of an actual rating being lower than the limit applied to power flows. Such a policy could use a certain low percentile from a probabilistic forecast and, for the case study discussed, overcomes the low wind speed problem. Furthermore, the possibility of large exceedences of actual ratings is removed by practical limitations on the degree of uprating.

Building on the present work, the proposed methods should be extended further to determine the percentiles of RTTR forecasts to apply to the whole OHL, using spatial interpolation methods to infer random weather samples experienced at all spans. The PIT histograms of RTTR forecasts for the entire OHL will be examined to determine whether the correlations among different weather variables or the same variables at different stations should be added into the random weather samples and which approach is most suited to estimation of ratings at the lower end of a distribution consistent with a system operator's risk policy. In addition, the 30-minute short-term or transient-state RTTR will be determined as the maximum current such that the maximum allowable conductor temperature is not exceeded given a current at that level for half-an-hour taking the initial temperature and thermal inertia of the overhead conductors into account. Further work will test the methods' performance over longer forecast time horizons.

\section{ACKNOWLEDGEMENTS}

Support for the work described in this paper is gratefully acknowledged from the Energy Technology Partnership, SP Energy Networks and National Grid Electricity Transmission (Network Innovation Allowance project NIA_NGET0105).

\section{REFERENCES}

[1] A. Michiorri, P.C. Taylor, S.C.E. Jupe, and C.J. Berry, "Investigation into the influence of environmental conditions on power system ratings," Proc. IMechE A: J. Power and Energy, vol. 223, pp. 743-757, Nov. 2009.

[2] T. Ringelband, P. Schafer, and A. Moser, "Probabilistic ampacity forecasting for overhead lines using weather forecast ensembles," Elect. Eng., vol. 95, issue 2, pp. 99-107, Jun. 2013.

[3] IEEE standard for calculating the current-temperature of bare overhead conductors, IEEE Std. 738, 2007.

[4] CIGRE Work. Group B2.43, "Guide for thermal rating calculations of overhead lines", Tech. Brochure 601, CIGRE, Paris, Dec. 2014.

[5] C.F. Price and R.R. Gibbon, "Statistical approach to thermal rating of overhead lines for power transmission and distribution," Proc. Inst. Electr. Eng. C - Gener. Transm. Distrib., vol. 130, issue 5, pp. 245-256, 1983. 
[6] S.C.E. Jupe, M. Bartlett, and K. Jackson, "Dynamic thermal ratings: the state of the art," in $21^{\text {st }}$ Int. Conf. on Electricity Distrib., CIRED, no. 918, pp. 1-4, Jun. 2011.

[7] B. Adam, "Weather-based and conductor state measurement methods applied for dynamic line rating forecasting," in 2011 Int. Conf. Advanced Power Syst. Automation and Protection, vol. 1, pp. 762-765.

[8] E. Fernandez, I. Albizu, G. Buigues, V. Valverde, A. Etxegarai, and J.G. Olazarri, "Dynamic line rating forecasting based on numerical weather prediction," in Proc. 2015 IEEE PowerTech Conf., pp. 1-6.

[9] J. Zhang, J. Pu, J.D. McCalley, H. Stern, and W.A. Gallus, “A Bayesian approach for short-term transmission line thermal overload risk assessment," IEEE Trans. Power Del., vol. 17, issue 3, pp. 770-778, Jul. 2002.

[10] D. Kim and J. Kim, "Prediction of transmission-line rating based on thermal overload probability using weather models," European Trans. Elect. Power, vol. 20, issue 4, pp. 534-544, 2010.

[11] V.T. Morgan, Thermal Behaviour of Electrical Conductors: Steady, Dynamic \& Fault-Current Ratings, Somerset, U.K.: Research Studies Press, 1991.

[12] Scottish Power Energy Networks, "Implementation of Real-Time Thermal Ratings", Final Close Down Rep., LCNF SPT1001, 2013.

[13] D.C. Hill, D. McMillan, K.R.W. Bell, and D. Infield, "Application of auto-regressive models to U.K. wind speed data for power system impact studies," IEEE Trans. Sustain. Energy, vol. 3, issue 1, pp. 134-141, Jan. 2012.

[14] J. Boland, "Time Series Modelling of Solar Radiation," in Modeling Solar Radiation at the Earth's Surface: Recent Advances, ch. 11, Springer-Verlag, 2008, pp. 283-312.

[15] G. Box, G. Jenkins, and G. Reinsel, Time Series Analysis: Forecasting and Control, $4^{\text {th }}$ Ed., New Jersey: John Wiley \& Sons, Inc., 2008.

[16] H. Lütkepohl, New Introduction to Multiple Time Series Analysis, New York: Springer-Verlag, 2005.

[17] F. Fan, K. Bell, and D. Infield, "Probabilistic weather forecasting for dynamic line rating studies," Accepted by $19^{\text {th }}$ Power Syst. Computation Conf., Genoa, 2016.

[18] T. Gneiting, K. Larson, K. Westrick, M.G. Genton, and E. Aldrich, "Calibrated probabilistic forecasting at the stateline wind energy center: the regime-switching space-time method," J. Amer. Statistical Assoc., vol. 101, pp. 968-979, Sep. 2006.

[19] F.X. Diebold, T.A. Gunther, and A.S. Tay, "Evaluating density forecasts with applications to financial risk management," in Symp. Forecasting and Empirical Methods in Macroeconomics and Finance, Int. Econ. Rev., vol. 39, pp. 863-883, Nov. 1998.

[20] E.P. Grimit, T. Gneiting, V.J. Berrocal, and N.A. Johnson, "The continuous ranked probability score for circular variables and its application to mesoscale forecast ensemble verification," Quart. J. Roy. Meteorological Soc., vol. 132, pp. 2925-2942, 2006.

[21] P. Berens, "CircStat: a MATLAB toolbox for circular statistics," $J$. Statistical Software, vol. 31, issue 10, pp. 1-21, Sep. 2009.

[22] T. Gneiting, A.E. Raftery, A.H. Westveld, and T. Goldman, "Calibrated probabilistic forecasting using ensemble model output statistics and minimum CRPS estimation," Monthly Weather Rev., vol. 133, issue 5, pp. 1098-1118, May 2005.

[23] H. Hersbach, "Decomposition of the continuous ranked probability score for ensemble prediction system," Weather and Forecasting, vol. 15, issue 5, pp. 559-570, Oct. 2000.

[24] T. Gneiting and A.E. Raftery, "Strictly proper scoring rules, prediction, and estimation," J. Amer. Statistical Assoc., vol. 102, issue 477, pp.359-378, 2007.

[25] S.A. Geer, "Least squares estimation," Encyclopedia of Statist. in Behavioural Science, vol. 2, pp. 1041-1045, 2005.

[26] R.Y. Rubinstein and D.P. Kroese, Simulation and the Monte Carlo method, $2^{\text {nd }}$ Ed., New Jersey: John Wiley \& Sons, Inc., 2007.

[27] The MathWorks Inc., normrnd, Normal Random Numbers. [Online]. Available: http://uk.mathworks.com/help/stats/normrnd.html, Accessed on: Sep. 29, 2015.
[28] B. Luong, Truncated Gaussian. (2010) [Online]. Available: http://uk.mat hworks.com/matlabcentral/fileexchange/23832-truncated-gaussian

[29] R.L. Iman and W.J. Conover, "A distribution-free approach to inducing rank correlation among input variables," Commun. in Statist. - Simulation and Computation, vol. 11, issue 3, pp. 311-334, 1982.

[30] T.A. Jones, "MATLAB functions to analyze directional (azimuthal) data II: Correlation," Comput. Geosci., vol. 36, issue 4, pp. 520-525, 2010.

[31] MATLAB Release 2012a, The MathWorks, Inc., Natick, Massachusetts, United States.

[32] F. Fan, K. Bell, D. Hill, and D. Infield, "Wind forecasting using kriging and vector auto-regressive models for dynamic line rating studies," in Proc. 2015 IEEE PowerTech Conf., pp. 1-6.

[33] J. Parkes and A. Tindal, "Forecasting short term wind farm production in complex terrain," in Proc. EWEC Conf., London, U.K., 2004.

[34] B.E. Hansen, "Lecture Notes on Nonparametrics," Uni. Wisconsin, 2009 [Online]. Available: http://www.ssc.wisc.edu/ bhansen/718/NonParamet rics1.pdf

[35] W. Wang and S. Pinter, "Dynamic Line Rating Systems for Transmission Lines", Topical Rep., U.S. Dept. of Energy, Apr. 2014.

[36] J.C. McCall and T. Goodwin, "Dynamic line rating as a means to enhance transmission grid resilience," in 2015 Grid of the Future Symp., CIGRE.

[37] "Engineering Recommendation P27: Current Rating Guide for High Voltage Overhead Lines Operating in the UK Distribution System," Energy Networks Assoc., 1986.

[38] CIGRE Work. Group B2.36, "Guide for application of direct real-time monitoring systems," Tech. Brochure 498, CIGRE, Paris, Jun. 2012.

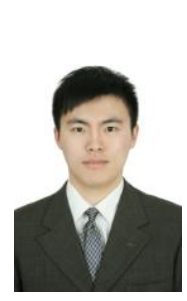

\section{BIOGRAPHIES}

Fulin Fan (S'15) is currently purchasing towards the Ph.D. degree at the University of Strathclyde, Glasgow, U.K. He received the B.Eng. in electronic and electrical engineering from both North China Electric Power University and University of Strathclyde, in 2012. His doctoral work is focused on estimating probabilistic forecasts of Real-Time Thermal Rating for overhead lines and investigating their applications.

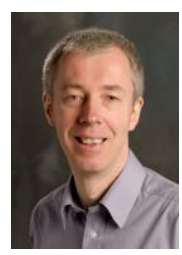

Keith Bell (M'13) is the ScottishPower Professor of Smart Grids at the University of Strathclyde. He joined the University in 2005 having previously gained his Ph.D. at the University of Bath and worked as an electrical engineering researcher in Bath, Manchester and Naples, and as a system development engineer in the electricity supply industry in Britain. He is a co-Director of the multi-disciplinary UK Energy Research Centre, an invited expert member of CIGRE Study Committee C1 on System Development and Economics and a member of the Council of the IET Power Academy, an initiative to promote electric power engineering as a graduate career in the U.K. He is a Chartered Engineer and has advised the Scottish Government, the Republic of Ireland government, the Northern Ireland Executive, Ofgem and the UK Department of Energy and Climate Change on electrical energy and power systems issues.

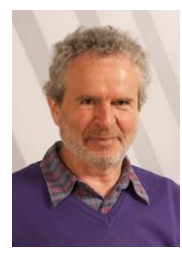

David Infield (M'04-SM'05) received a B.A. degree in mathematics and physics from the University of Lancaster, Lancaster, U.K. and the Ph.D. degree in applied mathematics from the University of Kent, Canterbury, U.K. He worked for the Rutherford Appleton Laboratory in Oxfordshire, U.K., from 1982 to 1993 researching into wind electricity systems. From 1993 to 2007, he was with Loughborough University, Leicestershire, U.K., where he established CREST, the Centre for Renewable Energy Systems Technology. He is now Professor of Renewable Energy Technologies with the Institute for Energy and Environment within the Department of Electronic and Electrical Engineering, University of Strathclyde, Glasgow U.K. Prof. Infield is Editor-in-Chief of the IET Journal Renewable Power Generation, and contributes to various IEC, CENELEC, and IPCC activities. 